\title{
Tissue Is the Issue in Transient Ischemic Attack and Stroke
}

$M$ ost of us were taught in medical school that a transient ischemic attack (TIA) was a stroke syndrome that resolved within 24 hours. With the advent of diffusion-weighted magnetic resonance imaging (DWMRI), several observers noted that although clinical symptoms had resolved in TIA patients, some had positive DW-MRI signal, implying infarcted brain. ${ }^{1}$ Because the overwhelming majority of TIAs resolve within 1 hour, and so many patients with symptoms lasting longer than several minutes have positive DW-MRI signal, a new definition of TIA was proposed a decade ago, which included brief focal neurologic symptoms referable to a vascular territory that did not have imaging signal implying infarction. ${ }^{2}$ Conversely, a new "tissue" definition of stroke, to distinguish it from TIA, has emerged to encompass clinical symptoms lasting $>24$ hours and/or evidence of infarcted brain at any time point, most frequently observed by DW-MRI. ${ }^{3}$

At the same time that we were wrestling with the question of when TIA ends and stroke begins, researchers were noting that patients with TIA had very high risk of having another event, this time a completed clinical stroke, within the first few days to a month after a clinical TIA. ${ }^{4}$ Important clinical tools to address subsequent stroke risk in patients presenting with clinical TIA were developed and promulgated. ${ }^{5}$ DW-MRI-positive signal is associated with a significant increase in the risk of subsequent short-term stroke risk. ${ }^{6}$ Most of the studies that have examined DW-MRI signal in clinical TIA were designed to investigate the predictive value of DW-MRI for short-term clinical stroke risk.

In the January issue of Annals of Neurology, Brazzelli et al performed a meta-analysis of these studies and point out the remarkable heterogeneity in the prevalence of DW-MRI signal in patients presenting with brief cerebrovascular symptoms. ${ }^{7}$ Whenever large variability is seen in the application of a test, in this case DW-MRI, one needs to question the utility of the test and what accounts for the variability. As the authors point out, even the notion that positive DW-MRI signal is permanent and synonymous with infarction has been questioned. ${ }^{8}$ The authors are correct to ponder the tremendous variability seen in the proportion of patients presenting with brief cerebrovascular symptoms and positive DW-MRI. We wonder, however, if some of this variability was introduced due to the papers available for their analysis being focused on a different question (Does positive DW-MRI predict shortterm stroke risk?), rather than the question of the metaanalysis (What is the frequency of positive DW-MRI in patients with brief cerebrovascular symptoms?). That is, the studies included were not designed to accurately estimate DW-MRI-positive prevalence among the source population. Most meta-analyses aggregate data from observational studies or clinical trials performed to answer the same question. ${ }^{9}$ Perhaps the only ideal way to study this would be to include all patients presenting with TIA symptoms from a community or a national randomly chosen sample and perform DW-MRI on all patients. However, the meta-analysis included some studies with consecutive patients and some community studies, and these still showed great variability.

Brazzelli et al's current, well-executed meta-analysis has synthesized the available information on DW-MRI prevalence. So what should we take away from it? It is true that in these particular studies, there is large heterogeneity in DW-MRI signal in clinical TIA. Recent publications suggesting that we use MRI to operationalize a tissue definition of TIA (absence of DW-MRI signal) and stroke (presence of DW-MRI signal) should consider and further investigate this heterogeneity before adopting new definitions for these conditions, which have been consistently defined for decades. Certainly, epidemiologic and clinical studies as well as national and international statistics that guide spending for research and care would be made more difficult if MRI is required in addition to clinical diagnoses. This may result in underestimation of stroke in populations without resources for MRI, such as developing countries. In brief, the current work says, not so fast, what does DW-MRI signal in clinical TIA really mean?

What the article does not alter is the evidence showing a clear link between positive DW-MRI signal among patients with brief cerebrovascular symptoms and the very high short term risk for ischemic stroke. ${ }^{5,6}$ In association with the rest of the $\mathrm{ABCD}^{2}$ risk algorithm, this information should lead to urgent evaluation and treatment of patients with very high short-term stroke risk. This includes rapid identification of patients with high-grade carotid artery disease and cardiac sources of emboli. Furthermore, the recent CHANCE trial results from China suggest the potential beneficial impact of 
clopidogrel loading combined with aspirin immediately after TIA to prevent stroke. ${ }^{10}$ The POINT trial, which is similar despite some important distinctions, including the more diverse patient population, is ongoing and will add to the CHANCE results. ${ }^{11}$

Brazzelli et al do an excellent job of making us pause to think about what this adolescent technology, DW-MRI, really means to the epidemiology of stroke and TIA. In a time when technology development has outpaced our ability to know when to apply technology advancements and when not to use new technology, we say bravo to them for reminding us that we should think before we leap.

\section{Potential Conflicts of Interest}

L.B.M. is a member of the Executive Committee of the NIH National Institute of Neurologic Disorders and Stroke-funded POINT trial mentioned in this editorial.

Lewis B. Morgenstern, $\mathrm{MD}^{1}$ and Brisa N. Sánchez, $\mathrm{PhD}^{2}$ ${ }^{1}$ Stroke Program, University of Michigan Medical School and Department of Epidemiology, University of Michigan School of Public Health, Ann Arbor, MI

${ }^{2}$ Department of Biostatistics, University of Michigan School of Public Health, Ann Arbor, MI

\section{References}

1. Crisostomo RA, Garcia MM, Tong DC. Detection of diffusionweighted MRI abnormalities in patients with transient ischemic attack: correlation with clinical characteristics. Stroke 2003;34:932-937.
2. Albers GW, Caplan LR, Easton JD, et al. Transient ischemic attack-proposal for a new definition. N Engl J Med 2002;347: 1713-1716.

3. Sacco RL, Kasner SE, Broderick JP, et al. An updated definition of stroke for the 21st century: a statement for healthcare professionals from the American Heart Association/American Stroke Association. Stroke 2013;44:2064-2089.

4. Johnston SC, Gress DR, Browner WS, Sidney S. Short-term prog nosis after emergency department diagnosis of TIA. JAMA 2000; 284:2901-2906.

5. Giles MF, Albers GW, Amarenco P, et al. Early stroke risk and $A B C D 2$ score performance in tissue- vs time-defined TIA: a multicenter study. Neurology 2011;77:1222-1228.

6. Giles MF, Albers GW, Amarenco $P$, et al. Addition of brain infarction to the ABCD2 score (ABCD2i): a collaborative analysis of unpublished data on 4574 patients. Stroke 2010;41:19071913.

7. Brazzelli M, Chappell F, Miranda H, et al. Diffusion-weighted imaging and diagnosis of transient ischemic attack. Ann Neurol 2014;75:67-76.

8. Peters JM, Maclean AV, Young GS. Rapid resolution of diffusion weighted MRI abnormality in a patient with a stuttering stroke. BMJ Case Rep 2010;2010.

9. Stroup DF, Berlin JA, Morton SC, et al. Meta-analysis of observational studies in epidemiology: a proposal for reporting. Metaanalysis Of Observational Studies in Epidemiology (MOOSE) group. JAMA 2000;283:2008-2012.

10. Wang $Y$, Zhao $X$, Liu $L$, et al. Clopidogrel with aspirin in acute minor stroke or transient ischemic attack. N Engl J Med 2013;369: 11-19.

11. Johnston SC, Easton JD, Farrant M, et al. Platelet-Oriented Inhibition in New TIA and minor ischemic stroke (POINT) trial: rationale and design. Int J Stroke 2013;8:479-483.

DOI: 10.1002/ana.24082 